\title{
Correction: Prediction and analysis of the modular structure of cytochrome P450 monooxygenases
}

Demet Sirim, Michael Widmann, Florian Wagner and Jürgen Pleiss

\section{Correction}

In our article (Sirim et al. BMC Struct Biol 2010, 10: 34) [1] the cytochrome P450 monooxygenase P450cam was referred to as CYP101D. In the latest release 3.0 of our cytochrome P450 database CYPED (www.CYPED.unistuttgart.de) P450cam was reassigned to family CYP101A and referred to as CYP101A1.

Received: 22 February 2012 Accepted: 25 April 2012

Published: 25 April 2012

\section{Reference}

1. Sirim D, Widmann M, Wagner F, Pleiss J: Prediction and analysis of the modular structure of cytochrome P450 monooxygenases. BMC Struct Biol 2010, 10:34.

doi:10.1186/1472-6807-12-4

Cite this article as: Sirim et al:: Correction: Prediction and analysis of the modular structure of cytochrome P450 monooxygenases. BMC Structural Biology 2012 12:4.

\footnotetext{
*Correspondence: juergen.pleiss@itb.uni-stuttgart.de

Institute of Technical Biochemistry, University of Stuttgart, Allmandring 31,

70569, Stuttgart, Germany
} 